Ambiente \& Água - An Interdisciplinary Journal of Applied Science
ISSN 1980-993X - doi:10.4136/1980-993X
www.ambi-agua.net
E-mail: ambi.agua@gmail.com

\title{
Análise de metodologias de quantificação de substâncias húmicas em lixiviados de aterros de resíduos sólidos
}

\author{
doi:10.4136/ambi-agua.1972
}

Received: 17 Jul. 2016; Accepted: 24 Nov. 2016

\author{
Letícia Sobral Maia S. Lima ${ }^{1}$; Ronei de Almeida ${ }^{1}$; \\ Bianca Ramalho Quintaes ${ }^{2}$; Daniele Maia Bila ${ }^{3}$; \\ Juacyara Carbonelli Campos ${ }^{1^{*}}$ \\ ${ }^{1}$ Universidade Federal do Rio de Janeiro (UFRJ), Rio de Janeiro, RJ, Brasil \\ Departamento de Processos Inorgânicos \\ ${ }^{2}$ Companhia Municipal de Limpeza Urbana (COMLURB), Rio de Janeiro, RJ, Brasil \\ Gerência de Pesquisas Aplicadas \\ ${ }^{3}$ Universidade do Estado do Rio de Janeiro (UERJ), Rio de Janeiro, RJ, Brasil \\ Departamento de Engenharia Sanitária e do Meio Ambiente (DESMA) \\ *Autor correspondente: e-mail: juacyara@eq.ufrj.br, \\ sobral@eq.ufrj.br, rnyalmeida@gmail.com, \\ biaquintaes@yahoo.com.br,danielebilauerj@gmail.com
}

\section{RESUMO}

Substâncias Húmicas (SH) são definidas como polímeros amorfos, de massa molar elevada, estruturas complexas e heterogêneas, que alteram com frequência as suas conformações em função das interações que ocorrem entre os grupos funcionais presentes na sua estrutura. A presença dessas substâncias principalmente nos lixiviados de aterros de resíduos sólidos e, em maior quantidade, em lixiviados de aterros antigos, dificulta o tratamento dos lixiviados, devido ao alto grau de recalcitrância de tal matéria orgânica. Nesse estudo, dois métodos de quantificação de SH foram testados em dois lixiviados gerados em aterros do Estado do Rio de Janeiro, oriundos do Aterro Controlado de Gericinó (ACG) e o Aterro Metropolitano de Gramacho (AMG), assim como o seu fracionamento nas frações Ácidos Húmicos (AH) e Ácidos Fúlvicos (AF). Os resultados de SH foram, respectivamente, 616 e $608 \mathrm{mg} \mathrm{L}^{-1}$ para o lixiviado proveniente do ACG e 1182 e $1191 \mathrm{mg} \mathrm{L}^{-1}$ para o lixiviado proveniente do AMG. O lixiviado oriundo do ACG apresentou 33\% (201 mg L $\left.{ }^{-1}\right)$ de AH e $67 \%$ (411 $\left.\mathrm{mg} \mathrm{L}^{-1}\right)$ de $\mathrm{AF}$ e o de AMG apresentou 37\% (437 $\left.\mathrm{mg} \mathrm{L}^{-1}\right)$ de $\mathrm{AH}$ e $63 \%$ (741 $\left.\mathrm{mg} \mathrm{L}^{-1}\right)$ de AF.

Palavras-chave: ácidos fúlvicos, ácidos húmicos, matéria orgânica recalcitrante.

\section{Analysis of quantification methodologies for humic substances in leachates from solid waste landfills}

\begin{abstract}
Humic substances (HS) are defined as amorphous polymers, with high molecular weight and complex and heterogeneous structures that frequently change their configurations depending on the interactions between the functional groups present in their structures. The presence of these substances, particularly in landfill leachates and in greater quantities in
\end{abstract}


leachates from old landfills, hinders the treatment of leachate due to the high degree of recalcitrance of organic matter. In this study, two quantification methods were tested for two leachates generated in solid waste landfills located in Rio de Janeiro State: the Gericinó Controlled Landfill Gericinó (GCL) and the Gramacho Metropolitan Landfill (GML). The study also analyzed the fractionation of humic substances in fractions of Humic acids (HA) and fulvic acids (FA). The results of the quantification of HS were, respectively, 616 and 608 $\mathrm{mg} \mathrm{L}^{-1}$ for the leachate from GCL and 1182 and $1191 \mathrm{mg} \mathrm{L}^{-1}$ for leachate from GML. The leachate from GCL presented $33 \%\left(201 \mathrm{mg} \mathrm{L}^{-1}\right)$ of HA and $67 \%\left(411 \mathrm{mg} \mathrm{L}^{-1}\right)$ of FA and the leachate from GML presented $37 \%\left(437 \mathrm{mg} \mathrm{L}^{-1}\right)$ of HA and $63 \%\left(741 \mathrm{mg} \mathrm{L}^{-1}\right)$ of FA.

Keywords: fulvic acids, humic acids, recalcitrant organic matter.

\section{INTRODUÇÃO}

A maioria das substâncias orgânicas presentes em aterros de resíduos é degradada na forma de gases compostos principalmente de metano $\left(\mathrm{CH}_{4}\right)$ e dióxido de carbono $\left(\mathrm{CO}_{2}\right)$, enquanto algumas substâncias orgânicas refratárias são estabilizadas nos aterros ou removidas dos mesmos sob a forma de lixiviado (Castilhos Jr., 2006). Em lixiviados de aterros jovens, a maior parte do carbono orgânico está presente como ácidos graxos voláteis. No entanto, em aterros maduros, substâncias húmicas $(\mathrm{SH})$ dominam a fração orgânica de lixiviados provenientes de aterros onde predomina a fase metanogênica em até 60\% (Šir et al., 2012).

As substâncias húmicas podem ser definidas como uma série de polímeros amorfos de coloração amarela-marrom a preta, de massa molar relativamente alta, formados por reações de oxidação e subsequente polimerização da matéria orgânica, durante o processo de decomposição de resíduos vegetais e animais presentes no ambiente (Brum, 2005). Apresentam-se como uma mistura heterogênea de moléculas polidispersas com elevadas massas molares e grupos funcionais distintos contendo oxigênio na forma de carboxilas, hidroxilas fenólicas e carbonilas (Christensen et al., 1998).

Os compostos orgânicos refratários encontrados nos lixiviados são compostos principalmente por substâncias húmicas, que incluem ácidos húmicos $(\mathrm{AH})$, ácidos fúlvicos (AF) e huminas (Kjeldsen et al., 2002).

As composições médias de unidades básicas para os ácidos húmicos e fúlvicos, em termos de fórmulas químicas, são respectivamente, $\mathrm{C}_{187} \mathrm{H}_{186} \mathrm{O}_{89} \mathrm{~N}_{9} \mathrm{~S}_{2}$ e $\mathrm{C}_{135} \mathrm{H}_{182} \mathrm{O}_{95} \mathrm{~N}_{5} \mathrm{~S}_{2}$ (Schnitzer e Khan, 1978). No nível estrutural, as três frações húmicas são análogas, diferindo na massa molar e na quantidade de grupos funcionais (Kawahigashi, 2012).

As SH interagem facilmente com espécies metálicas e alguns compostos orgânicos, podendo aumentar a solubilidade de compostos hidrofóbicos e solubilizar metais através de complexação, modificando a biodisponibilidade e biotoxicidade de compostos poluentes presentes nos aterros (Christensen et al., 1998).

Numerosos instrumentos analíticos e abordagens têm sido aplicadas à análise de $\mathrm{SH}$, incluindo a espectroscopia de fluorescência (Antunes e Silva, 2005), de ressonância magnética nuclear (Peuravuori e Pihlaja, 2003), cromatografia de exclusão de tamanho de alto desempenho (Chin et al., 1994) e de espectrometria de massa (Mugo et al., 2007). Essas metodologias são sensíveis e precisas, contudo são métodos com maior custo operacional e que levam mais tempo em operação que métodos que utilizam avaliação espectrofotométrica, o que aumenta a necessidade de se buscar metodologias sensíveis, viáveis economicamente e de fácil execução para a análise quantitativa das substâncias húmicas do lixiviado, principalmente para empresas que tratam os lixiviados.

Um dos principais problemas relacionados ao gerenciamento de resíduos sólidos é o tratamento efetivo dos lixiviados produzidos nos aterros, visto que essa matriz é complexa e 
contém elevada concentração de matéria orgânica recalcitrante de difícil degradação: as substâncias húmicas. Nesse contexto, se faz necessário o estudo de metodologias de quantificação, identificação e remoção das SH para avaliar a eficiência dos tratamentos convencionais.

A recalcitrância da matéria orgânica e a cor adquirida nos lixiviados de aterros de resíduos tornam a quantificação de substâncias húmicas uma importante etapa de caracterização para a escolha do método mais adequado de tratamento de lixiviado.

Assim, este estudo teve como objetivo avaliar a utilização de dois métodos de quantificação de SH mais simples que os instrumentos analíticos que já são utilizados, e seu posterior fracionamento, e aplicá-los a dois lixiviados de diferentes origens, provenientes do Aterro Controlado de Gericinó (ACG) e do Aterro Metropolitano de Gramacho (AMG), localizados no Estado do Rio de Janeiro.

\section{MATERIAIS E MÉTODOS}

\subsection{Lixiviados em estudo}

Neste trabalho foram utilizados lixiviados de dois diferentes aterros do Estado do Rio de Janeiro: o Aterro Controlado de Gericinó (ACG) e o Aterro Metropolitano de Gramacho (AMG). No período de três anos, entre 2012 e 2015, foram coletadas seis amostras de cada lixiviado nas lagoas de estabilização localizadas nos próprios aterros.

O Aterro Controlado de Gericinó, situado no bairro de Bangu, no município do Rio de Janeiro, começou suas operações em 1987, como vazadouro a céu aberto. Recebia, em média, 2000 toneladas de resíduos sólidos urbanos por dia, gerando cerca de $500 \mathrm{~m}^{3} \mathrm{~d}^{-1}$ de lixiviado, armazenados na lagoa de acumulação existente (Costa et al., 2015). O ACG estava em fase de fechamento durante a coleta das amostras, em 2014.

O mais antigo dentre os aterros selecionados, o Aterro Metropolitano de Gramacho, está situado no município de Duque de Caxias. Segundo Campos et al. (2013), o aterro, aberto em 1978 operou como um lixão, teve suas atividades encerradas em junho de 2012, recebendo durante quase 35 anos a maior parte dos resíduos sólidos do município do Rio de Janeiro, ou seja, cerca de 9500 t de resíduos por dia. Ainda segundo os autores, mesmo após o encerramento do aterro, ele ainda produz, a cada dia, entre $1000 \mathrm{~m}^{3}$ e $2000 \mathrm{~m}^{3}$ de lixiviado.

São duas matrizes diferenciadas, visto que o lixiviado produzido no ACG apresenta contribuição de um aterro antigo (27 anos), fechado, que recebeu apenas resíduos urbanos e, o lixiviado produzido no AMG, apresenta contribuição de um aterro antigo (35 anos), fechado, que recebeu resíduo urbano, químico hospitalar e industrial (Campos et al., 2013) enquanto estava em operação.

A caracterização dos lixiviados foi realizada com base nos seguintes parâmetros físico-químicos, suas respectivas metodologias analíticas e equipamentos (APHA, 2005): Absorvância em $254 \mathrm{~nm}$ - Abs 254, que fornece uma indicação do conteúdo de matéria orgânica aromática (Método 5910-B, Shimadzu UV mini 1240), Carbono Orgânico Total - COT (Método 5310-C, TOC Analyzer - Hipertoc 1000), Cor (Método 2120-C, Espectrofotômetro DR2800 e reator Hach), Demanda Química de Oxigênio - DQO (Método 5220-D, Espectrofotômetro DR2800 e reator Hach) e pH (Método 4500-B, pHmetro microprocessador Quimis).

\subsection{Quantificação de Substâncias Húmicas}

\section{Método de Sheng Modificado}

O método espectrofotométrico/colorimétrico original foi desenvolvido por Sheng et al. (2007) e aplicado para determinar a concentração de substâncias húmicas em águas naturais. 
Š́r et al. (2012) modificaram essa metodologia a fim de quantificar as substâncias húmicas presentes em lixiviados provenientes de aterros de resíduos. O método de Sheng modificado se baseia na ligação do corante azul de toluidina (AT) a moléculas de ácidos húmicos para produzir um complexo que provoca a diminuição da absorbância a $603 \mathrm{~nm}$. A fórmula molecular do AT é $\mathrm{C}_{15} \mathrm{H}_{16} \mathrm{ClN}_{3} \mathrm{~S}$ e sua fórmula estrutural é ilustrada na Figura 1.<smiles></smiles>

Figura 1. Fórmula estrutural do corante Azul de Toluidina (AT). Fonte: Sheng et al. (2007).

Aos tubos de vidro de $10 \mathrm{~mL}$ foram adicionados $1 \mathrm{~mL}$ da solução de AT 1,5 mmol L $\mathrm{m}^{-1}$, 2,5 mL da solução de citrato-fosfato de sódio $0,05 \mathrm{~mol} \mathrm{~L}^{-1}(\mathrm{pH} 7,0)$ e $0,5 \mathrm{~mL}$ da solução de EDTA $0,05 \mathrm{~mol} \mathrm{~L}^{-1}$, esse último reagente visando à remoção de cátions potencialmente interferentes (por exemplo: $\mathrm{Ca}^{2+}, \mathrm{Mg}^{2+} \mathrm{e} \mathrm{Fe}^{3+}$ ). Em seguida, $4 \mathrm{~mL}$ de água destilada ou de amostras de lixiviado foram adicionados, e os tubos foram deixados à temperatura ambiente por 20 min antes da medição de absorbância, em espectrofotômetro (HACH), em $603 \mathrm{~nm}$. A água destilada foi utilizada como referência.

A concentração de SH em função da absorbância foi determinada com base na equação da reta de linearização da curva padrão para $\mathrm{SH}$, empregando padrões de $20,40,60 \mathrm{mg} \mathrm{L}^{-1}$, preparados com ácido húmico comercial, a partir da solução estoque de concentração $2 \mathrm{~g} \mathrm{~L}^{-1}$, pH 8,0, armazenada a $4^{\circ} \mathrm{C}$, no escuro. $\mathrm{O}$ cálculo para determinação de $\mathrm{SH}$, foi realizado utilizando-se a Equação 1.

$$
\Delta \mathrm{A}=\mathrm{A}_{0}-\mathrm{A}
$$

em que:

$\Delta \mathrm{A}=$ Absorbância real da amostra;

$\mathrm{A}=$ Absorbância do ensaio com amostra adicionada; $\mathrm{e}$

$\mathrm{A}_{0}=$ Absorbância do ensaio em branco de reagente, ou seja, com água destilada.

Foram realizados ensaios com a adição de padrão interno (Pi) preparado com ácido húmico comercial - AHc - (Sigma Aldrich), nas amostras de lixiviado e nas mesmas concentrações utilizadas na curva padrão de $\mathrm{SH}$ : 20, 40, $60 \mathrm{mg} \mathrm{L}^{-1}$. As amostras de lixiviados foram diluídas 10 e 20 vezes em água destilada de modo a ajustar à curva padrão.

Foi calculada a recuperação (Equação 2) para avaliar a quantidade medida da substância em relação à quantidade adicionada na matriz (ou branco), em um determinado número de testes (Burns et al., 2002).

$$
\mathrm{R}(\%)=\left(\mathrm{C}_{1}-\mathrm{C}_{2}\right) / \mathrm{C}_{3} \times 100
$$

em que:

$$
\begin{aligned}
& \mathrm{R}=\text { Recuperação }(\%) ; \\
& \mathrm{C}_{1}=\text { concentração na amostra adicionada (lixiviado + padrão); } \\
& \mathrm{C}_{2}=\text { concentração na amostra não adicionada (lixiviado); e }
\end{aligned}
$$




$$
\mathrm{C}_{3}=\text { concentração adicionada (padrão) }
$$

\section{Método de Lowry Modificado}

A determinação da concentração de substâncias húmicas nas amostras de lixiviado também foi realizada utilizando-se metodologia baseada no método de Lowry para proteínas (Frolund et al., 1995). Os autores modificaram essa metodologia a fim de quantificar as substâncias húmicas presentes em lodos biológicos. Essa modificação foi possível pois as substâncias húmicas, assim como as proteínas, reagem com o reagente Folin utilizado reduzindo-o, devido aos grupos fenólicos presentes. O método consiste na execução do método de Lowry com e sem a adição de $\mathrm{CuSO}_{4}$, em que a interferência da cor no ensaio sem a adição de $\mathrm{CuSO}_{4}$ é atribuída principalmente às substâncias húmicas (Moravia et al., 2011).

O princípio do método baseia-se em uma mistura contendo molibdato, tungstato e ácido fosfórico (reagente Folin Ciocalteau), que sofre uma redução ao reagir com proteínas, em meio alcalino e na presença do catalisador $\mathrm{Cu}^{+2}$, produzindo um composto azul escuro, com absorção máxima de $750 \mathrm{~nm}$. A absorbância desse composto, obtido após a redução do reagente de Folin, pode ser medida a $750 \mathrm{~nm}$, com elevada sensibilidade para baixas concentrações de substâncias húmicas, ou a $550 \mathrm{~nm}$, com baixa sensibilidade para elevadas concentrações de substâncias húmicas. Nesse procedimento, são utilizadas seis soluções:

- A (20g Na $\mathrm{CO}_{3}$ e 4g NaOH em 1000 mL $\mathrm{H}_{2} \mathrm{O}$ destilada);

- B (2,0g $\mathrm{CuSO}_{4} .5 \mathrm{H}_{2} \mathrm{O}$ em $100 \mathrm{~mL} \mathrm{H}_{2} \mathrm{O}$ destilada);

- $\mathrm{C}\left(2,0 \mathrm{~g} \mathrm{KNaC}_{4} \mathrm{H}_{4} \mathrm{O}_{6} \cdot 4 \mathrm{H}_{2} \mathrm{O}\right.$ em $100 \mathrm{~mL}$ de $\mathrm{H}_{2} \mathrm{O}$ destilada);

- D com cobre" (1 mL da solução B, $1 \mathrm{~mL}$ da solução C e $98 \mathrm{~mL}$ da solução A);

- D sem cobre"'(1 mL da solução C e 99 mL da solução A); e

- Folin $1 \mathrm{~mol} \mathrm{~L}^{-1}$ (diluir o reagente Folin-Ciocalteau na proporção 1:2 com água destilada).

Para se determinar o teor de SH foi diluída, em 10 vezes, a amostra de lixiviado em água destilada (volume utilizado $0,5 \mathrm{~mL}$ ) de modo a ajustar à curva padrão. Em seguida, foram adicionados $5 \mathrm{~mL}$ de solução "D com cobre" em cada tubo de ensaio para os ensaios com cobre; e $5 \mathrm{~mL}$ de solução "D sem cobre" para os ensaios sem cobre, e os tubos permaneceram em repouso por 10 minutos à temperatura ambiente.

Posteriormente, acrescentou-se 0,5 mL da solução de Folin 1:2 e, passados 30 minutos, foi feita a leitura das absorbâncias no espectrofotômetro nos comprimentos de onda $550 \mathrm{~nm}$, para concentração de $\mathrm{SH}$ maior que $25 \mathrm{mg} \mathrm{L}^{-1}$, e $750 \mathrm{~nm}$ para concentração de $\mathrm{SH}$ na faixa de 5 a $25 \mathrm{mg} \mathrm{L}^{-1}$.

Visando à adequação do ensaio para o efluente em estudo, a concentração de substâncias húmicas em função da absorbância foi determinada com base na reta de linearização da curva padrão para $\mathrm{SH}$, empregando padrões de $0,17,52$ e $101 \mathrm{mg} \mathrm{L}^{-1}$, preparados com ácido húmico comercial (Moravia et al., 2011). Em seguida, é calculada a absorbância dissociada ou corrigida de SH para cada amostra com cobre e sem cobre, pelo uso da Equação 3.

$$
A_{S H}=\left(A_{s / C u}-\left(F \times A_{c / C u}\right)\right) /(1-F)
$$

em que:

$\mathrm{A}_{\mathrm{SH}}=$ Absorbância para SH;

$\mathrm{F}=$ Fator de redução de absorbância $(\mathrm{F}=0,12$, nesse estudo);

$\mathrm{A}_{\mathrm{s} / \mathrm{Cu}}=$ absorbância sem a adição $\mathrm{CuSO}_{4} ; \mathrm{e}$ 
$\mathrm{A}_{\mathrm{c} / \mathrm{Cu}}=$ absorbância com a adição $\mathrm{CuSO}_{4}$.

O Fator de redução de absorbância (F) é calculado pelo uso da Equação 4.

$$
\mathrm{F}=\left(\mathrm{A}_{\mathrm{s} / \mathrm{Cu}}\right) /\left(\mathrm{A}_{\mathrm{c} / \mathrm{Cu}}\right)
$$

Foi utilizado a adição de padrão interno (Pi) preparado com ácido húmico comercial, nas mesmas concentrações utilizadas na curva padrão de SH: $17,51,102 \mathrm{mg} \mathrm{L}^{-1}$. As amostras de lixiviados foram diluídas 10 e 20 vezes em água destilada de modo à ajustar a curva padrão.

A recuperação foi calculada segundo a Equação 2 (Burns et al., 2002).

\subsection{Fracionamento de substâncias húmicas}

Com o objetivo de investigar as características da matéria orgânica recalcitrante presente em lixiviados de aterros de resíduos e o quanto essas substâncias recalcitrantes contribuem para a sua complexidade, foi realizado o fracionamento das substâncias húmicas.

A matéria orgânica recalcitrante presente no lixiviado foi separada em frações $\mathrm{AH}, \mathrm{AF}$ e Hyi (fração Não Húmica) usando o método descrito por Christensen et al. (1998). A fração $\mathrm{AH}$ foi precipitada por ajuste do $\mathrm{pH}$ do lixiviado para inferior a $1 \mathrm{e}$, em seguida, foi separada por centrifugação (Q222TM104, Quimis) a 50 rpm durante 5 minutos e filtração através de uma membrana de nitrato de celulose de 0,45 $\mu \mathrm{m}$ (Sartorius Stedim). Depois, o AH foi redissolvido em $0,05 \mathrm{~mol} \mathrm{~L}^{-1}$ de $\mathrm{NaOH}$.

A resina XAD-8 (Supelco Analytical) foi utilizada para reter o AF por adsorção. O AF retido foi separado por eluição com uma solução de $0,1 \mathrm{~mol} \mathrm{~L}^{-1}$ de $\mathrm{NaOH}$. A matéria orgânica dissolvida residual foi considerada como fração hidrofílica (Hyi) ou não húmica. As concentrações das frações AH e AF foram calculadas utilizando o método de quantificação de Sheng modificado, pois este é mais simples de execução e apresenta menor tempo operacional.

\section{RESULTADOS E DISCUSSÃO}

\subsection{Caracterização físico-química dos lixiviados em estudo}

A Tabela 1 ilustra os resultados da caracterização físico-química dos lixiviados dos ACG e AMG utilizados neste estudo.

Tabela 1. Caracterização físico-química dos lixiviados provenientes dos ACG e AMG (n=6).

\begin{tabular}{|c|c|c|c|c|c|c|}
\hline \multirow{2}{*}{ Parâmetros } & \multicolumn{3}{|c|}{ ACG } & \multicolumn{3}{|c|}{ AMG } \\
\hline & Mínimo & Máximo & Média & Mínimo & Máximo & Média \\
\hline Absorbância 254 nm & 11 & 21 & 14 & 21 & 30 & 25 \\
\hline Cor $\left(\mathrm{mgPtCo} \mathrm{L}{ }^{-1}\right)$ & 4977 & 5679 & 5241 & 5643 & 8197 & 7332 \\
\hline $\mathrm{COT}\left(\mathrm{mgC} \mathrm{L}^{-1}\right)$ & 437 & 648 & 555 & 815 & 1297 & 972 \\
\hline $\mathrm{DQO}\left(\mathrm{mgO}_{2} \mathrm{~L}^{-1}\right)$ & 978 & 1813 & 1515 & 1776 & 2194 & 1998 \\
\hline $\mathrm{pH}$ & 7,7 & 8,6 & 8,1 & 7,9 & 8,8 & 8,3 \\
\hline
\end{tabular}

Pode-se observar que embora haja variação nas características dos lixiviados, a concentração de matéria orgânica é elevada, o pH está na faixa básica e há presença de matéria orgânica aromática. A absorbância em $254 \mathrm{~nm}$ tem sido usada para avaliar a remoção de compostos orgânicos em processos de tratamento de águas e efluentes (APHA, 2005).

Segundo Castilho Jr. et al. (2006), as concentrações de DQO tendem a sofrer reduções ao longo da degradação dos resíduos aterrados. Entretanto, essa redução pode ser lenta devido à presença de elevadas concentrações de matéria orgânica de difícil degradação. Ainda segundo 
os autores, essas são características comuns em lixiviados mais estabilizados, provenientes de aterros mais antigos, como é o caso dos aterros estudados, que possuem maior quantidade de compostos recalcitrantes, que resistem à biodegradação e tendem a persistir e se acumular no ambiente.

\subsection{Avaliação da Concentração das Substâncias Húmicas}

A curva padrão de $\mathrm{AH}$ obteve um coeficiente de correlação $\left(\mathrm{R}^{2}\right)$ de 0,999 . A concentração de SH em função da absorbância medida foi determinada com base na equação da reta de linearização da curva padrão para SH para o método de Sheng Modificado (Equação 5).

$$
\mathrm{SH}=(\Delta \mathrm{A}-0,2327) / 0,0009
$$

em que:

$\mathrm{SH}=$ concentração de $\mathrm{SH}\left(\mathrm{mg} \mathrm{L}^{-1}\right)$; e

$\Delta \mathrm{A}=$ absorbância real da amostra.

A Tabela 2 ilustra os resultados de recuperação do método de Sheng modificado e a concentração de SH presentes nos lixiviados oriundos dos aterros ACG e AMG.

As amostras de lixiviados foram diluídas 10 vezes e 20 vezes, em água destilada, de modo a se ajustarem à curva padrão. Em menores (5 vezes) ou maiores diluições (40 vezes) a concentração de substâncias húmicas não se ajustou à curva padrão construída, ou seja, nessas diluições a concentração de substâncias húmicas é muito alta ou muito baixa para ser corretamente medida pelo método. As diluições foram necessárias para ajustar a concentração das SH à faixa linear da curva de calibração.

O lixiviado gerado no AMG apresenta concentrações de $\mathrm{SH}$ mais elevadas do que o lixiviado gerado no ACG porque o AMG recebeu resíduos urbanos e industriais, enquanto estava em operação. Dentro deste contexto, Zamora e Morais (2005), Souto e Povinelli (2007), Foo e Hameed (2009) afirmam que a composição e a concentração de contaminantes nos lixiviados são influenciadas pelo tipo de resíduos descartados e pela idade do aterro. Os autores enfatizam ainda que um lixiviado cuja composição deriva de um aterro maduro é mais complexo que um lixiviado composto a partir de uma nova operação de descarga, apresentando elevadas concentrações de ácidos húmicos e fúlvicos e de baixa biodegradabilidade.

A Tabela 3 ilustra os resultados de recuperação do método de Lowry Modificado e a concentração de $\mathrm{SH}$ presentes nos lixiviados oriundos dos aterros ACG e AMG.

Os resultados de concentração de SH mostrados na Tabela 4, para diluição de 20 vezes, tem desvio padrão mais elevado do que os resultados para a diluição de 10 vezes, tanto Gericinó $(0,98 \%$ e $1,96 \%)$ como para Gramacho $(4,187 \%$ e 5,08\%). Todos os resultados de recuperação estão dentro de um intervalo aceitável. O lixiviado oriundo do Aterro Metropolitano de Gramacho apresenta concentrações de SH mais elevadas que o lixiviado do aterro de Gericinó, $1191 \mathrm{mg} \mathrm{L}^{-1}$ e $607,5 \mathrm{mg} \mathrm{L}^{-1}$, respectivamente, o que era esperado devido às características dos resíduos dispostos nos aterros.

Os resultados de quantificação de SH obtidos para os dois diferentes métodos avaliados foram bem semelhantes (Tabelas 2 e 3). Isto mostra que os métodos, apesar de se basearem em diferentes princípios, são adequados para a análise quantitativa de $\mathrm{SH}$ presentes nos lixiviados em estudo. Além disso, são metodologias sensíveis, econômicas e de fácil execução, mostrando-se viáveis no gerenciamento de aterros e estações de tratamento de lixiviados.

As principais limitações operacionais dos métodos avaliados são relativas ao intervalo de 
validade da curva-padrão construída com a solução de ácido húmico da Sigma Aldrich. Para o método de Sheng é de 0 a $60 \mathrm{mg} \mathrm{L}^{-1}$ de ácido húmico e para o método de Lowry, até $100 \mathrm{mg} \mathrm{L}^{-1}$ de ácido húmico. A vantagem do método de Sheng é que apresenta etapas mais simples e menor tempo de execução.

Tabela 2. Concentração de SH nas amostras dos lixiviados oriundos dos aterros de ACG e AMG, pelo método Sheng modificado $(\mathrm{n}=6)$.

\begin{tabular}{|c|c|c|c|}
\hline Identificação das Amostras & $\mathrm{SH}\left(\mathrm{mg} \mathrm{L}^{-1}\right)$ & Desvio* $(\%)$ & Recuperação (\%) \\
\hline ACG Diluída 10x & 614,00 & 5,15 & - \\
\hline ACG Diluída 10x + 20 mgAHc L ${ }^{-1}$ & 634,44 & 1,72 & 102 \\
\hline ACG Diluída 10x + 40 mgAHc L ${ }^{-1}$ & 653,33 & 4,01 & 98 \\
\hline ACG Diluída 10x + 60 mgAHc L ${ }^{-1}$ & 673,33 & 3,42 & 99 \\
\hline ACG Diluída 20x & 618,00 & 7,57 & - \\
\hline ACG Diluída $20 x+20$ mgAHc L ${ }^{1}$ & 637,78 & 3,36 & 99 \\
\hline ACG Diluída 20x + 40 mgAHc L ${ }^{-1}$ & 655,56 & 3,81 & 94 \\
\hline ACG Diluída $20 x+60$ mgAHc L ${ }^{-1}$ & 677,78 & 6,72 & 100 \\
\hline AMG Diluída 10x & 1181,00 & 1,10 & - \\
\hline AMG Diluída $10 x+20$ mgAHc L ${ }^{-1}$ & 1202,11 & 2,91 & 105 \\
\hline AMG Diluída $10 x+40$ mgAHc $L^{-1}$ & 1220,00 & 2,29 & 98 \\
\hline AMG Diluída 10x + 60 mgAHc L ${ }^{-1}$ & 1242,22 & 1,27 & 102 \\
\hline AMG Diluída 20x & 1184,00 & 2,54 & - \\
\hline AMG Diluída $20 x+20$ mgAHc L ${ }^{-1}$ & 1205,44 & 3,36 & 102 \\
\hline AMG Diluída $20 x+40$ mgAHc $\mathrm{L}^{-1}$ & 1222,22 & 5,54 & 96 \\
\hline AMG Diluída 20x + 60 mgAHc L ${ }^{-1}$ & 1244,00 & 5,08 & 100 \\
\hline
\end{tabular}

Nota: Desvio $=\left(\sum(\text { valor }- \text { média dos valores })^{2} /(\text { número de amostras }-1)\right)^{1 / 2}$.

$\mathrm{AHc}=$ ácido húmico comercial.

Para o método de Sheng modificado, os resultados de recuperação estão dentro de um intervalo acima de $90 \%$. Os desvios padrão apresentam valores relativamente baixos, e esse comportamento repetiu-se para as diferentes diluições (10x e 20x) e para as diferentes concentrações de padrões internos utilizadas, o que aumenta a confiabilidade no método e nos resultados.

Para o método de Lowry modificado, a curva padrão de AH obteve um coeficiente de correlação de 0,9998. A concentração de SH em função da absorbância foi determinada com base na equação da reta de linearização da curva padrão para SH (Equação 6).

$$
\mathrm{SH}=10 \times(\mathrm{A}-0,0056) / 0,0006
$$

em que:

$\mathrm{SH}=$ concentração de $\mathrm{SH}\left(\mathrm{mg} \mathrm{L}^{-1}\right)$; e

$\mathrm{A}=$ absorbância da amostra. 
Tabela 3. Concentração de SH nas amostras dos lixiviados oriundos dos aterros de ACG e AMG, pelo método Lowry modificado $(\mathrm{n}=6)$.

\begin{tabular}{|c|c|c|c|}
\hline Identificação das Amostras & $\mathrm{SH}\left(\mathrm{mg} \mathrm{L}^{-1}\right)$ & Desvio* $(\%)$ & Recuperação (\%) \\
\hline ACG Diluída 10x & 608,00 & 0,98 & - \\
\hline ACG Diluída 10x + 20 mgAHc L ${ }^{-1}$ & 626,74 & 3,48 & 94 \\
\hline ACG Diluída 10x + 40 mgAHc L ${ }^{-1}$ & 647,76 & 3,43 & 99 \\
\hline ACG Diluída 10x + 60 mgAHc L ${ }^{-1}$ & 667,27 & 0,06 & 99 \\
\hline ACG Diluída 20x & 607,00 & 1,96 & - \\
\hline ACG Diluída $20 x+20$ mgAHc L ${ }^{1}$ & 631,96 & 3,93 & 125 \\
\hline ACG Diluída 20x + 40 mgAHc L ${ }^{-1}$ & 649,69 & 5,08 & 107 \\
\hline ACG Diluída 20x + 60 mgAHc L ${ }^{-1}$ & 670,53 & 6,89 & 106 \\
\hline AMG Diluída 10x & 1192,00 & 4,18 & - \\
\hline AMG Diluída $10 x+20$ mgAHc $L^{-1}$ & 1213,48 & 5,10 & 107 \\
\hline AMG Diluída 10x + 40 mgAHc L $\mathrm{L}^{-1}$ & 1232,80 & 4,18 & 102 \\
\hline AMG Diluída 10x + 60 mgAHc L ${ }^{-1}$ & 1252,50 & 4,38 & 101 \\
\hline AMG Diluída 20x & 1190,00 & 5,08 & - \\
\hline AMG Diluída 20x + 20 mgAHc L ${ }^{-1}$ & 1212,12 & 6,89 & 111 \\
\hline AMG Diluída $20 x+40$ mgAHc $L^{-1}$ & 1234,47 & 6,97 & 111 \\
\hline AMG Diluída $20 x+60$ mgAHc $L^{-1}$ & 1251,13 & 6,60 & 102 \\
\hline
\end{tabular}

Nota: Desvio $=\left(\sum(\text { valor }- \text { média dos valores })^{2} /(\text { número de amostras }-1)\right)^{1 / 2}$.

$\mathrm{AHc}=$ ácido húmico comercial.

\subsection{Fracionamento de Substâncias Húmicas}

Os resultados do fracionamento das substâncias húmicas presentes nos lixiviados estudados encontram-se na Tabela 4. Ressalta-se que as frações de AH e AF nos lixiviados em estudo foram quantificadas apenas pelo método de Sheng modificado, sendo esse método de execução mais rápida quando comparado ao método de Lowry modificado.

Tabela 4. Fracionamento das $\mathrm{SH}$ dos lixiviados provenientes dos aterros ACG e AMG (n=6).

\begin{tabular}{lrr}
\hline Fração & ACG & AMG \\
\hline $\mathrm{SH}\left(\mathrm{mg} \mathrm{L}^{-1}\right)$ & 616 & 1182 \\
$\mathrm{AH}\left(\mathrm{mg} \mathrm{L}^{-1}\right)$ & $201(33 \%)$ & $437(37 \%)$ \\
$\mathrm{AF}\left(\mathrm{mg} \mathrm{L}^{-1}\right)$ & $411(67 \%)$ & $741(63 \%)$ \\
\hline
\end{tabular}

A caracterização das SH e suas frações em amostras de lixiviado de diferentes origens pode facilitar o desenvolvimento de uma capacidade de previsão do comportamento das SH de acordo com a idade do aterro.

Em estudos realizados por Christensen et al. (1998), os autores concluíram que, da fração de matéria orgânica recalcitrante, os ácidos húmicos e fúlvicos são responsáveis por aproximadamente 40 e $60 \%$, respectivamente. Essa afirmação foi confirmada em estudos 
posteriores realizados por Huo et al. (2008), onde os autores encontraram concentrações de 30 a $40 \%$ de $\mathrm{AH}$ e de 60 a $70 \%$ de $\mathrm{AF}$ em lixiviados estabilizados, provenientes de aterros maduros. Esses resultados estão de acordo com os obtidos no presente trabalho.

A investigação das características da matéria orgânica presente em lixiviados de aterros de resíduos nas formas de frações húmicas e fúlvicas pode auxiliar a entender melhor o nível de recalcitrância presente no lixiviado: quanto maior a preponderância de substâncias húmicas em relação às fúlvicas, mais resistente à biodegradação será o lixiviado. A partir dessa informação, consegue-se estabelecer um sistema de tratamento de lixiviado mais adequado.

\section{CONCLUSÕES}

Os resultados de quantificação de SH nos lixiviados, com os métodos de Sheng e Lowry modificados, respectivamente, foram $616 \mathrm{mg} \mathrm{L}^{-1}$ e $608 \mathrm{mg} \mathrm{L}^{-1}$ para Gericinó e $1182 \mathrm{mg} \mathrm{L}^{-1} \mathrm{e}$ $1191 \mathrm{mg} \mathrm{L}^{-1}$ para Gramacho, mostrando 1,4\% de desvio para aterro de Gericinó e 0,72\% para o aterro de Gramacho.

Os métodos utilizados neste estudo são simples, rápidos e sensíveis, tem baixo consumo de amostra, são economicamente viáveis e de fácil execução para a análise quantitativa de SH em lixiviados. Os resultados de recuperação foram superiores a 90\% para os lixiviados em estudo. Estes fatores dão confiabilidade na utilização desses métodos de quantificação.

Em relação às frações húmicas encontradas, para ambos aterros, a fração $\mathrm{AH}$ variou na faixa de $33-37 \%$, enquanto que a fração fúlvica variou na faixa de 63-67\%.

\section{REFERÊNCIAS}

AMERICAN PUBLIC HEALTH ASSOCIATION - APHA. Standard Methods for the Examination of Water and Wastewater. 21. ed. Washington, 2005.

ANTUNES, M. C. G.; SILVA, J. Multivariate curve resolution analysis excitation-emission matrices of fluorescence of humic substances, Analytica Chimica Acta, v. 546, n. 1, p. 52-59, 2005. http://dx.doi.org/10.1016/j.aca.2005.05.003

BRUM, M. C. Remoção de ácido húmico da água por precipitação e flotação com a utilização de surfatantes catiônicos. 2005. 61f. Dissertação (Mestrado em Engenharia Metalúrgica e de Materiais) - COPPE, Universidade Federal do Rio de Janeiro, Rio de Janeiro, 2005.

BURNS, D. T.; DANZER, K.; TOWNSHEND, A. Use of the term "recovery" and "apparent recovery" in analytical procedures. Pure and Applied Chemistry, v. 74, n. 11, p. 2201-2205, 2002. https://doi.org/10.1351/pac200274112201

CAMPOS, J. C.; MOURA, D.; COSTA, A. P.; YOKOYAMA, L.; ARAUJO, F. V. F.; CAMMAROTA, M. C. Evaluation of $\mathrm{pH}$, alkalinity and temperature during air stripping process for ammonia removal from landfill leachate. Journal of Environmental Science and Health, Part A: Toxic/Hazardous Substances and Environmental Engineering, v. 48, n. 9, p. 1105-1113, 2013. http://dx.doi.org/10.1080/10934529.2013.774658

CASTILHOS JR., A. B. Gerenciamento de resíduos sólidos urbanos com ênfase na proteção de corpos d'água: prevenção, geração e tratamento de lixiviados de aterros sanitário. In: CASTILHOS JR., A. B. (Org.). Resíduos sólidos urbanos: aterro sustentável para municípios de pequeno porte. Rio de Janeiro: Rima/ABES, 2006. p. 494. 
CHIN, Y. P.; GSCHWEND, P. M. The abundance, distribution, and configuration of porewater organic colloids in recent sediments. Geochimica et Cosmochimica Acta, v. 55, n. 5, p. 1309-1317, 1994. http://dx.doi.org/10.1016/0016-7037(91)90309-S

CHRISTENSEN, J. B.; JENSEN, D. L.; GRON, C.; FILIP, Z.; CHRISTENSEN, T. H. Characterization of the dissolved organic carbon in landfill leachate-polluted groundwater. Water Research, v. 32, n. 1, p. 125-135, 1998. http://dx.doi.org/10.1016/S0043-1354(97)00202-9

COSTA, F. M.; CAMPOS, J. C.; FONSECA, F. V.; BILA, D. M. Tratamento de lixiviados de aterros de resíduos sólidos utilizando Processos Fenton e Foto-Fenton Solar. Revista Ambiente \& Água, v. 10, n. 1, p. 109-110, 2015. http://dx.doi.org/10.4136/ambiagua. 1483

FROLUND, B.; GRIEBE, T.; NIELSEN, P. H. Enzymatic activity in the activated-sludge floc matrix. Applied Microbiology and Biotechnology, v. 43, n. 4, p. 755 - 761, 1995.

FOO, K. Y.; HAMEED, B. H. An overview of landfill leachate treatment via activated carbon adsorption process. The Journal of Hazardous Materials, v. 171, p. 54-60, 2009. http://dx.doi.org/10.1016/j.jhazmat.2009.06.038

HUO, S.; XI, B.; YU, H; HE, L.; FAN, S.; LIU, H. Characteristics of dissolved organic matter (DOM) in leachate with diferent landfill ages. Journal of Environmental Sciences, v. 20, n. 4, p. 492-498, 2008. http://dx.doi.org/10.1016/S1001-742(08)62085-9

KAWAHIGASHI, F. Aplicabilidade do pós-tratamento de lixiviado de aterro sanitário por adsorção em carvão ativado granular e avaliação ecotoxicológica. 2012. 155p. Dissertação (Mestrado de Edificações e Saneamento) - Centro de Tecnologia e Urbanismos, Universidade Estadual de Londrina, Londrina, 2012.

KJELDSEN, P. I.; BARLAZ, M. A.; ROOKER, A. P.; BAUN, A.; LEDIN, A.; CHRISTENSEN, T. H. Present and long-term composition of MSW landfill leachate: a review. Environmental Science and Technology, v. 32, n. 4, p. 297-336, 2002. http://dx.doi.org/10.1080/10643380290813462

MORAVIA, W. G.; LANGE, L. C.; AMARAL, M. C. S. Avaliação de processo oxidativos avançado pelo reagente de Fenton em condições otimizadas no tratamento de lixiviado de aterro sanitário com ênfase em parâmetros coletivos e caracterização do lodo gerado. Química Nova, v. 34, n. 8, p. 1370-1377, 2011. http://dx.doi.org/10.1590/S010040422011000800014

MUGO, S.; GETHI, M.; OKURO, J.; BERGVINSON, D.; GROOTE, H. D.; SONGA, J. Evaluation of stem borer resistant maize open pollinated varieties and hybrids on-station and on-farm in the moist mid-altitude maize ecologies of Kenya. In: AFRICAN CROP SCIENCE SOCIETY CONFERENCE, 8., 2007, El-Minia. Papers... El-Minia: Minia University, 2007. p. 959-964

PEURAVUORI, J. P.; PIHLAJA, K. Critical comments on accuracy of quantitative determination of natural humic matter by solid state CNMR spectroscopy. Talanta, v. 59, n. 1, p. 177-189, 2003. http://dx.doi.org/10.1016/S0039-9140(02)00476-9

SCHNITZER, M.; KHAN, S. U. Soil organic matter. Amsterdam: Elsevier, 1978. 319p. 
SHENG, G. P.; ZHANG, M. L.; YU, H. Q. A rapid quantitative method for humic substances determination in natural waters. Analytica Chimica Acta, v. 592, n. 2, p. 162-167, 2007. http://dx.doi.org/10.1016/j.aca.2007.04.024

ŠIR, M.; PODHOLA, M.; PATOCKA, T.; HONZAJKOVA, Z.; KOCUREK, P.; KUBAL, M. et al. The effect of humic acids on the reverse osmosis treatment of hazardous landfill leachate. Journal of Hazardous Materials, v. 207, p. 86-90, 2012. http://dx.doi.org/10.1016/j.jhazmat.2011.08.079

SOUTO, G. B.; POVINELLI, J. Varacterísticas do lixiviado de aterros sanitários no Brasil. In: CONGRESSO BRASILEIRO DE ENGENHARIA SANITÁRIA E AMBIENTAL, 24., 2007, Belo Horizonte. Anais... Belo Horizonte: ABES, 2007.

TATSI, A. A.; ZOUBOULIS, A. I.; MATIS, K. A.; SAMARAS, P. Coagulation-flocculation pre-treatment of sanitary landfill lichgates. Chemosphere, v. 53, p. 737-744, 2003. http://dx.doi.org/10.1016/S0045-6535(03)00513-7

TRAN, N. H.; NGO, H. H.; URASE, T.; GIN, K. Y. A critical review on characterization strategies of organic matter for wastewater and water treatment processes. Bioresource Technology, v. 193, p. 523-533, 2015. http://dx.doi.org/10.1016/j.biortech.2015.06.091

ZAMORA, P.; MORAIS, J. L. Use of advanced oxidation processes to improve the biodegradability of mature landfill leachates. Journal of Hazardous Materials, v. 123, n. 1/3, p. 181-186, 2005. http://dx.doi.org/10.1016/j.jhazmat.2005.03.041

ZOUBOULIS, A. I.; CHAI, X.; KATSOYIANNIS, I. A. The application of bioflocculant for the removal of humic acids from stabilized landfill leachates. Journal of Environmental Management, v. 70, n. 1, p. 35-41, 2004. http://dx.doi. org/10.1016/j.jenvman.2003.10.003 